\title{
Routing Discovery Algorithm Using Parallel Chase Packet
}

\author{
Muneer Bani Yassein \\ Dept. Computer Science \\ Jordan University of Science and Technology \\ Irbid, Jordan \\ Amera Al-Ameri \\ Dept. Computer Science \\ Jordan University of Science and Technology \\ Irbid, Jordan
}

\author{
Yaser M. Khamayseh \\ Dept. Computer Science \\ Jordan University of Science \\ and Technology \\ Irbid, Jordan \\ Wail E. Mardini \\ Dept. Computer Science \\ Jordan University of Science \\ and Technology \\ Irbid, Jordan
}

\begin{abstract}
On demand routing protocols for ad hoc networks such as Ad Hoc On Demand Distance Vector (AODV) initiate a route discovery process when a route is needed by flooding the network with a route request packet. The route discovery process in such protocols depends on a simple flooding as a broadcast technique due to its simplicity. Simple flooding results in packet congestion, route request overhead and excessive collisions, namely broadcast storm problem. A number of routing techniques have been proposed to control the simple flooding technique. Ideally, the broadcast of route request or the route discovery process must be stopped as soon as the destination node is found. This will free the network from many redundant packets that may cause network collision and contention.

In this paper, chasing packet technique is used with standard AODV routing protocol to end the fulfilled route requests. The chase packet is initiated by the source node and is broadcasted in parallel with route request packet. As soon as the destination is found the chase packet starts its work by trying to catch and discard the route request in early stages before it broadcasts further in the network.

Performance evaluation is conducted using simulation to investigate the performance of the proposed scheme against the existing approach that uses chase packet technique such as Traffic Locality Route Discovery Algorithm with Chase (TLRDA-C). Results reveal that the proposed scheme minimizes end-to-end packet delays and achieves low routing request overhead.
\end{abstract}

Keywords-MANET; Chase Packets; AODV; Broadcast Storm Problem.

\section{INTRODUCTION}

A computer network is a collection of independent devices interconnected together with the aid of some communication facilities. Wired networks were useful but not suitable for mobile environments. The production and popularity of mobile devices (such as laptops, and mobile phones) increased the interest in wireless networks, and increased the need to adopt changes in the communication ways $[2,12,13]$.

IEEE 802.11 standard defines two different modes for wireless network: infrastructure and infrastructure-less. The later is commonly known as Mobile Ad Hoc Network (MANET)? Infrastructure mode consists of a control unit called base station or access point and a number of mobile and/or fixed stations (nodes) [12]. The base station is responsible for managing and controlling the communication between the mobile stations as well as providing the connections to wired stations. MANET consists of a collection of distributed nodes that communicate with each other over a wireless medium using multi-hop communication techniques without the need of the base station [2].

The process of transmitting data from a source to a destination node in the network is called routing. During this process, one or more intermediate nodes cooperate to transfer the data. Routing involves two main tasks: first, determining the best path from the source to the destination node. Second task is transmitting data packets between the nodes [3]. Flooding is the simplest broadcast technique used to transmit the packet to the destination. It means that every node in the network receives the packet and rebroadcasts it to all its neighbors. Flooding consumes network resources and leads to low network delivery ratio [4].

According to the literature, there are many proposed schemes to alleviate the effects of conventional flooding, control the broadcast technique to cover part of the network and improve network performance in terms of overhead and congestion levels. They have been classified to the following four categories: Time-To-live (TTL), chase packets, location, and neighbor's information $[5,6,7,8]$.

In this paper we propose a scheme that uses chasing packet with standard AODV routing protocol to stop the fulfilled route request. The chase packet is initiated by the source node and broadcasted in parallel with route request packet. As soon as the destination is found the chase packet starts its work by trying to catch and discard the route request in early stages before it broadcasts further in the network. The rest of this paper is organized as follows. Section 2 provides some algorithms that are related to our work. Section 3 describes and illustrates the idea of the proposed scheme. 
Simulations results are analyzed and evaluated against existing routing protocol are provided in section 4. Finally, we conclude the paper in section 5 .

\section{RELATED WORK}

Chase packet based schemes broadcast control packets (called chase packet) to stop the continuous propagation of route requests once a path is discovered [2]. Limited Broadcasting algorithm (L-B) [6] uses the chase packet strategy to control the broadcast process. In this scheme, the source nodes commence a route request procedure using traditional broadcast mechanism. Once the path is found and an acknowledgement is received by the source, it commences an intercepting process to stop the search. L-B divides the network into two channels which means that the time slots will be divided into periods.

The time slots are divided among the two channels to match their given speeds. For example, if the given speed for channel 1 is $1 / 4$, and the given speed for channel 2 is $3 / 4$, then one fourth of the time slots in each period are assigned to channel 1, and the rest of the time slots are assigned to channel 2 . Typically, the route requests use channel one which means that it will use only $1 / 4$ of the channel time. This wills slowdown the route request propagation while the rest of the channel time is used to transmit route replies and broadcast chase packets. Therefore, the chase packets are three times faster than route requests. This will give the chase packets a chance to catch the fulfilled route requests $[2,11]$. The main deficiency of the L-B algorithm is favoring the chase packets and route replies over the route requests from the beginning. Route requests are delayed from the beginning before discovering the needed route which would delay all route discoveries.

Limited-Hop Broadcast Algorithm (LHBA) proposed in [9] overcomes the shortcoming in L-B algorithm by allowing any node that discovers the route to initiate the chase packet. The finder will broadcast the chase packet to $K$ hop neighbors to stop the further broadcast of the route requests. This algorithm allows any route finder or the destination itself to initiate the chase packet. Therefore, many chase packets will be initiated for the same route request [2].

Blocking- Expanding Ring Search (B-ERS) is an improvement of the Expanding Ring Search (ERS) proposed in [5] where each new ring starts from the previous ring instead of starting from the source node as in ERS. B-ERS introduces a delay equals $2 *$ hop count at each node within the ring. Two stop signals can be used to control the flooding of route request RREQ. One is the reply packet RREP which can be sent by any route node and the other is the chase packet which is called stop_instruction, it is sent by the source node only [5].

The RREP message informs that the destination is found. After the $2^{*}$ hop count units of time delay, the intermediate nodes in the current ring may receive a chase packet from the source node.
Stop instruction packet broadcasts to cover the current ring only where the finder of the route is located. Once the chase packet is received, the intermediate node will discard both chase and request packets. If no chase packet is received within the $2^{*}$ hop count units of time, this means that there is no node in this ring having any route information then a new ring will be initiated.

The Traffic Locality Oriented Route Discovery Algorithm with Chase Packet (TLRDA-C) proposed in [2] divides the network into two regions: First, neighborhood region which includes the most likely destination for the source node. Second, beyond-neighborhood region. Each node in neighborhood region will broadcast the RREQ packet without adding any extra delays. This will improve the route request discovery process. However, in beyond-neighborhood region, the route request is further broadcasted with a deliberate additional delay.

Once the source node receives the reply packet (RREP), it broadcasts chase packet to terminate the route request. The chase packet is broadcast without adding any delay in an effort to terminate the propagation of the fulfilled route request as soon as possible. The catching occurs in the beyondneighborhood region as the chase packet travels faster than its associated route request within this region; the route request is subject to a slight delay while propagating in this region [2]. The route discovery process may be delayed when the destination is located out of neighborhood region. TLRDA-C assumes that the route finder, F, is not located near the boundaries of the network [1]. In the case of source node mobility, it needs to re- initiate its neighborhood region $[2,13]$.

\section{Ad Hoc On Demand Distance Vector with PARAllel Chase PACKet (AODV-PC)}

This paper presents a modified scheme that utilizes the AODV routing protocol with chase packet concept. Instead of delay the route request when it reaches the beyondneighborhood region, the modified scheme broadcasts the route request using the same speed in any network region. It broadcast the chase packet parallel with the route request packet. The default state of the chase packet is an inactive state. When the destination is found, it will change the chase packet state into an active state and broadcast it to inform the other intermediate nodes that the route request should be stopped [11].

The chance to catch and discard the route request before covering a large area is high. The chase packet is very close to the route request when the destination is found. The chase packet is broadcasted without adding any delays similar to the route request to terminate the propagation of the fulfilled route request as soon as possible. Figure 1 shows the chase packet format used in the proposed scheme. Packet size should be chosen carefully because the transmitting and receiving operations consume bandwidth and power in wireless networks [1]. 


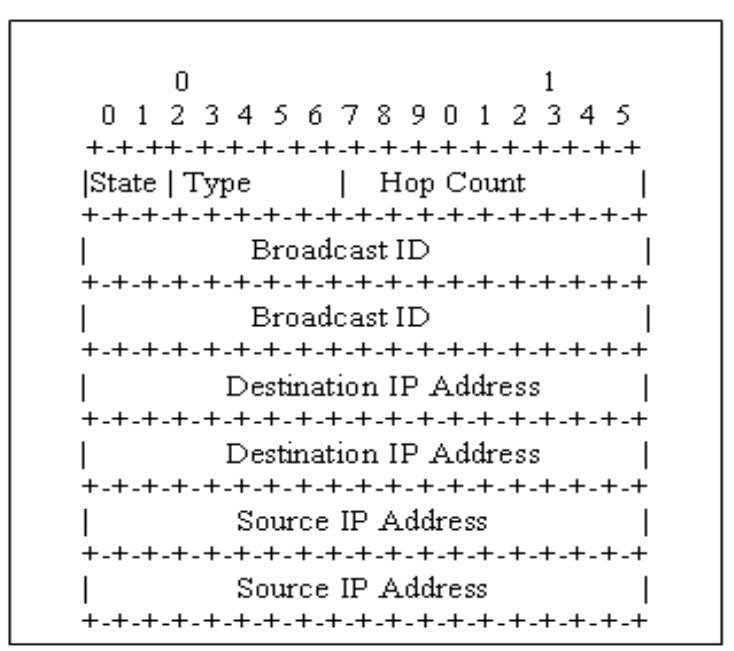

Fig.1. Chase packet format

In MANETs, the packets cross multiple nodes. Using small packets is more efficient in order to minimize resources consumption. So, chase packets in the proposed scheme are kept small in size, 14 bytes, compared to a route request packet where its sizes in TLRDA-C [2] and AODV [10] are 25 and 24 bytes respectively.

Figure 2 indicates the steps that are performed by each node when receiving the route request packet. The first step is to discard any duplicated route requests (line 2).

If the route request received for the first time (line 3 ), the node searches for the stored information in order to match chase packet. If it is found (line 4), the route request will be discarded after storing the needed information (lines 5-6). If no matching chase packet is received, the route request is processed according to the AODV routing protocol (line 8) [1].

\section{Steps preformed by each node upon receiving the chase packet in AODV-PC}

\section{1: If the chase is a duplicate}

\section{2: $\quad$ Discard it}

3: Else

4: If the current node is the destination

5: $\quad$ change the chase packet state to active

6: broadcast the chase packet

7: $\quad$ Else

8: If chase state is active then

9: $\quad$ insert into list

10 broadcast the chase packet

11: $\quad$ Else

12: $\quad$ broadcast the chase packet

$\begin{array}{ll}\text { 13: } & \text { End IF } \\ \text { 14: } & \text { End IF } \\ \text { 15: } & \text { End IF }\end{array}$

Fig.2. Processing of route requests at a node in AODV-PC

Once the chase packet is received, the steps in Figure 3 are executed at each node. The request is discarded by the node if the chase packet is a duplicate, (line 2). Otherwise, if the receiving node is the destination node, it changes the state of the chase packet to be active (line 5). Active state means that the chase packet starts its work and tries to discard the associated route request packet. If the receiving node is a normal intermediate node and the state of the chase packet is active then the node inserts the chase packet to the active chase packet list (line 9). This guarantees that if the chase packet is received before the request packet, it will do its work and discard the incoming route request packet [1].

In AODV-PC, the source node is always the initiator of the chase packets. This enables AODV-PC to avoid initiating many chase packets for the same route request.

\begin{tabular}{l} 
Steps preformed by each node upon receiving a route \\
request in AODV-PC \\
\hline \hline 1: If route request is a duplicate \\
2: Discard the route request \\
3: Else \\
4: If active chase packet has been received then \\
5: $\quad$ Store route request information \\
6: Discard the route request \\
7: $\quad$ Else \\
8: Continue according to standard AODV protocol \\
9: $\quad$ End IF \\
10: \\
\hline
\end{tabular}

Fig.3. Processing chase packet at node in AODV-PC

\section{Simulation Results AND AnAlysis}

In order to compare our results to other related works we used a simulation model that is similar to ones used in the literature. The simulation model consists of the following main components: Mobile nodes with specific transmission range in specific area. The typical values are transmission range up to $250 \mathrm{~m}$ in a square area of $600 \mathrm{~m} \times 600 \mathrm{~m}$.

The number of nodes will be varied for different simulation experiments. The simulation time used is 600 seconds. The IEEE 802.11 is used as the underlying MAC layer communication model. Packet generation rate is 4packet/second. Table 1 summarizes the main simulation parameters used. 
The following performance metrics has been used in order to evaluate the performance of our technique [12]:

- Packet loss: Which is defined as the total number of dropped packets in the whole network. The main factors affecting and causing packet loss are congestion and mobility.

- Route request overhead: This is defined as the total number of route requests received in all nodes in the network. Some request might not be satisfied and those sending new request will cause this metric to increase indicating network low performance.

- End-to-end delay: This metric includes all times from the time the packet was ready to be sent at the source node and the time it reaches the destination node. It includes all time delays due to route discovery, queuing, and propagation delay.

- Route request latency: This is usually defined as the average delays per hop among all route requests in a single simulation scenario. Latency of one route request is the average delay experienced by the route request per hop from the time it was sent by a source node until the time it was discarded by the chase packet which is usually called the Rout Request Life time RRL [2].

In the following subsections we study the effect of the network density and the mobility on the network performance using the above metrics.

\section{A. The Effect of Network Density}

Figs. 4 to 7 display the performance results for TLRDA-C versus AODV-PC using networks with different densities. The number of nodes increased from 20 to 100 in multiples on 20. The nodes speed is varying from a minimum of $1 \mathrm{~m} / \mathrm{s}$ and a maximum speed of $15 \mathrm{~m} / \mathrm{s}$. The number of the traffic load is four. The end-to-end delay increases with the network density for both algorithms because the hop count of transmission path increases which in turn increases the delay including the discovery time for the route.

TABLE I. PARAMETERS USED IN SIMULATION

\begin{tabular}{|l|l|}
\hline \multicolumn{1}{|c|}{ Parameters } & \multicolumn{1}{c|}{ Value } \\
\hline Simulator & NS2.33 \\
\hline Transmission Range & $250 \mathrm{~m}$ \\
\hline Network Size & $600 \mathrm{~m}$ x600m \\
\hline Simulation time & $900 \mathrm{~s}$ \\
\hline Packet Size & 512 byte \\
\hline Packet Rate & 4 pkt/s \\
\hline Traffic Type & Constant Bit Rate (CBR) \\
\hline Routing Protocol & AODV \\
\hline Number of Nodes & $20,40,60,80,100$ \\
\hline Number of runs per point & 20 \\
\hline MAC protocol & IEEE 802.11 \\
\hline Minimum speed & $1 \mathrm{~m} / \mathrm{s}$ \\
\hline Maximum speed & $2,5,7,10,13$ m/s \\
\hline Pause time & $50 \mathrm{~s}$ \\
\hline Mobility model & Random WayPoint model (RWP) \\
\hline
\end{tabular}

\section{End To End Delay}

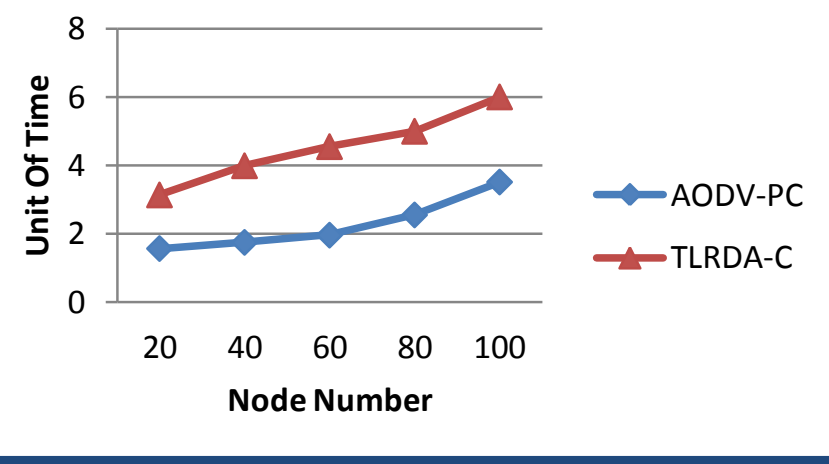

Fig.4. End-to-End delay versus network density

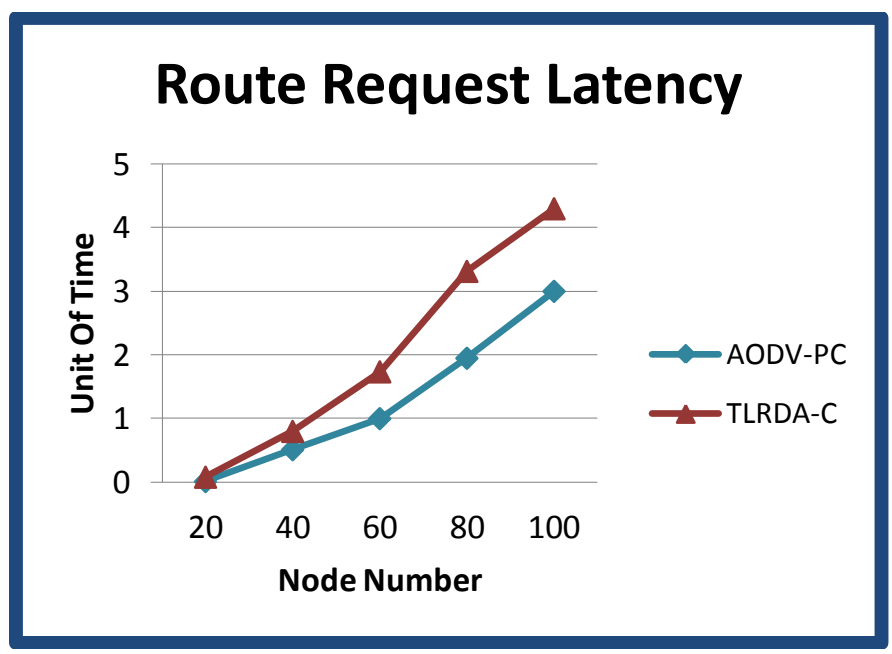

Fig.5. Average route request latency versus network density

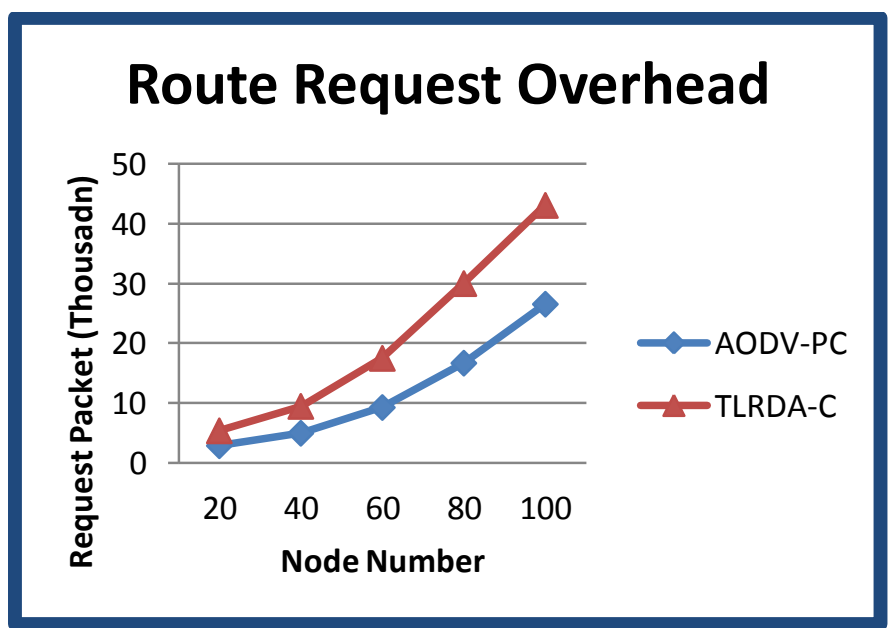

Fig.6. Average route request latency versus network density

AODV-PC improves the end to end delay. TLRDA-C algorithm performs well when the destination or the route finder locates at the neighborhood region. Otherwise, route request will be delayed to give the chase packet a chance to catch it. This will delay the route discovery process and increases the end to end delay. 


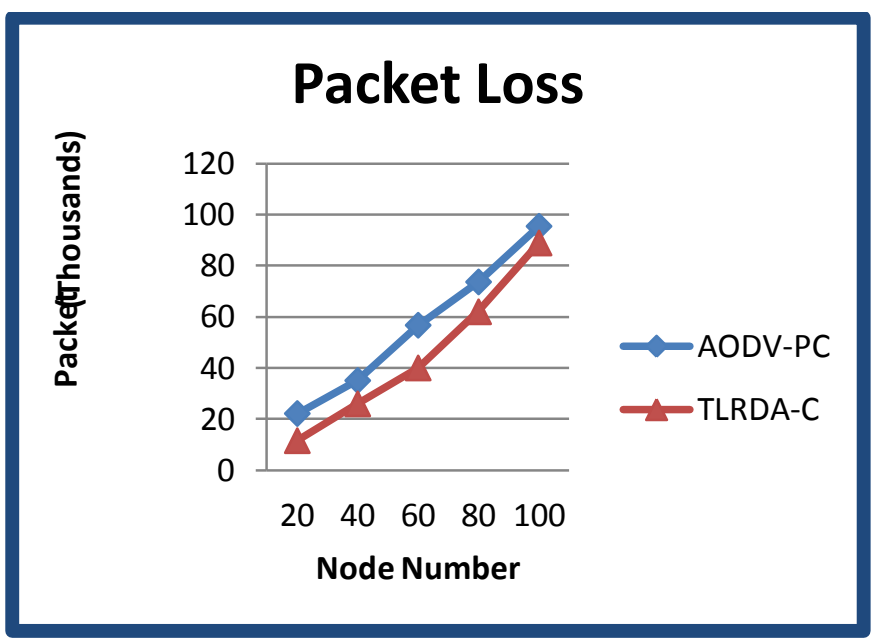

Fig.7. Packet loss versus network density

AODV-PC improves the average of route request latency in high density network. When the network density is 20 nodes the value of latency is approximately equal. This is due to the chosen scenario at that point; it means that the destination node may be in the neighborhood region. Therefore, the TLRDA-C algorithm performs well and catches the route request in early stages which is in turn reduces the latency.

The route request may reach more nodes each time it propagates further in the network and catching process may be difficult. The success of the catching process by the chase packet frees the network from more fulfilled route requests which improves the overhead. The main factors that have a high impact on the packet loss are mobility and congestion. Although our proposed scheme is less congests in general, but the TLRAD-C algorithm has less number of control packet such as chase packet, too many small chase packet in AODVPC algorithm may cause a high congestion in the area that is located between the source and destination where the data packet will be transmitted.

\section{B. The Effect of Mobility}

Figs. 8 to 11 display the effect of mobility on our metrics. The results are extracted from simulating both algorithms using networks of size 60 nodes. We use six different maximum speeds where the actual speed is randomly selected from [1, max speed]. The six maximum speeds take the following values: $2,5,7,10$, and $13 \mathrm{~m} / \mathrm{s}$ respectively. The traffic load was fixed to be 4 .

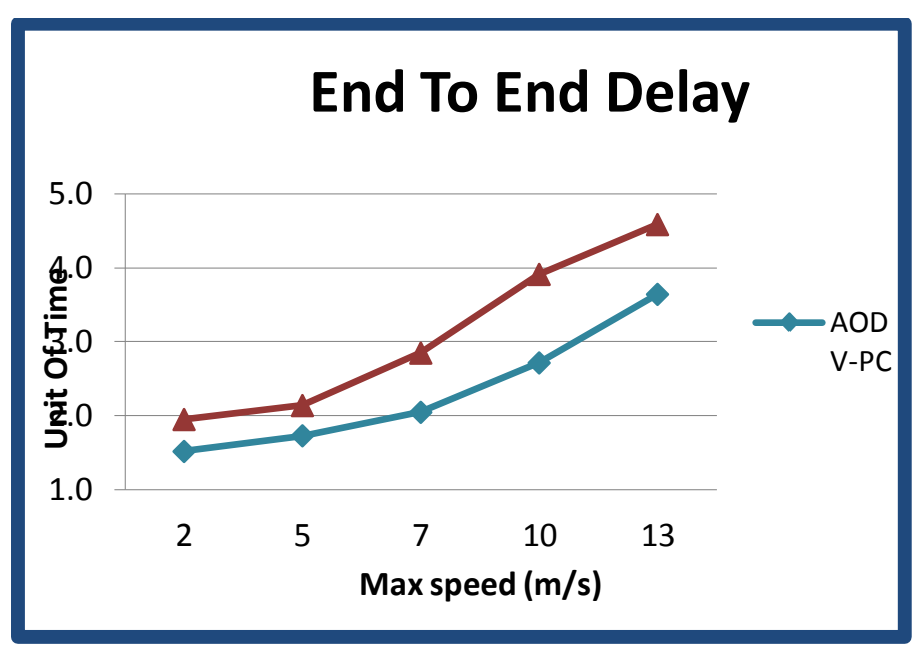

Fig.8. End-to-end delay versus maximum speed

AODV-PC offers better end-to-end delay as shown in Fig.8. This improvement is due to less congested environment provided by AODV-PC algorithm and quick broadcasting within the network regardless of the region.

The route request latency increases with the increment of speed due to the link breakage regardless of used algorithm as shown in Fig.9. AODV-PC reduces the route request latency this is due to the quick broadcasting within the network regardless of the region and due to better success rate ( The ability to discard route request in early stages) achieved by proposed algorithm. TLRDA-C algorithm is very depends on the used scenario which specifies the destination location.

Fig.10 demonstrates the route request overhead. As we mentioned before many route request will be reinitiated because of high network link breakage the network success rate has a high impact on the route request overhead. Lower number of redundant hop count (number of hops that the route request passes before it is discarded by the chase packet) means that better catching for the route request in early stages. This will free the network from many route requests and reduce its overhead.

Fig. 11 explores the effect of node speed on the packet loss. We can easily notice that the value of packet loss on both algorithms is approximately equal in highly mobile network. This is due to high congestion in the area located in between source and destination caused by the chase packet. High mobility increases the probability of link breakage which increases the packet loss. 


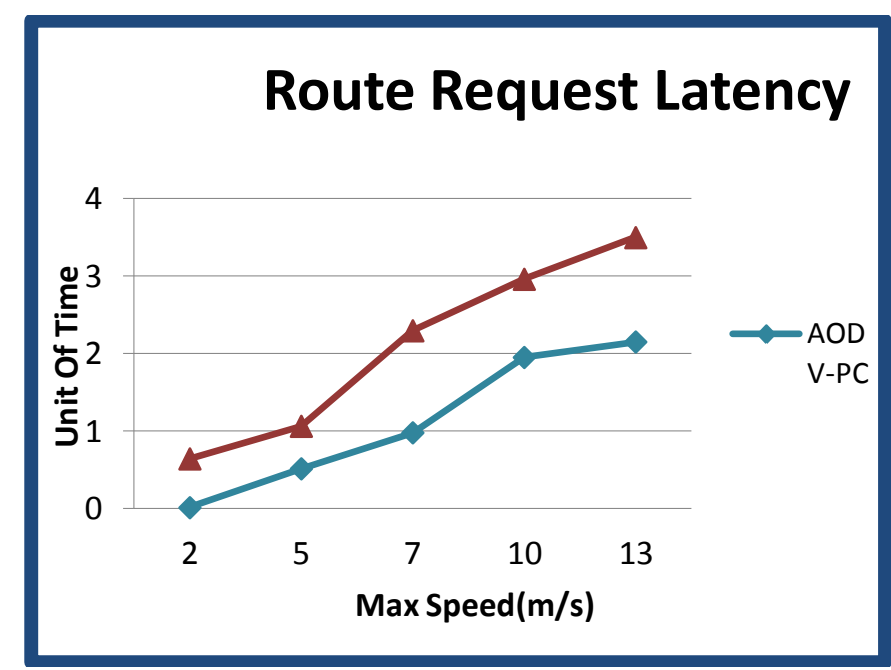

Fig.9. Route request latency versus maximum speed

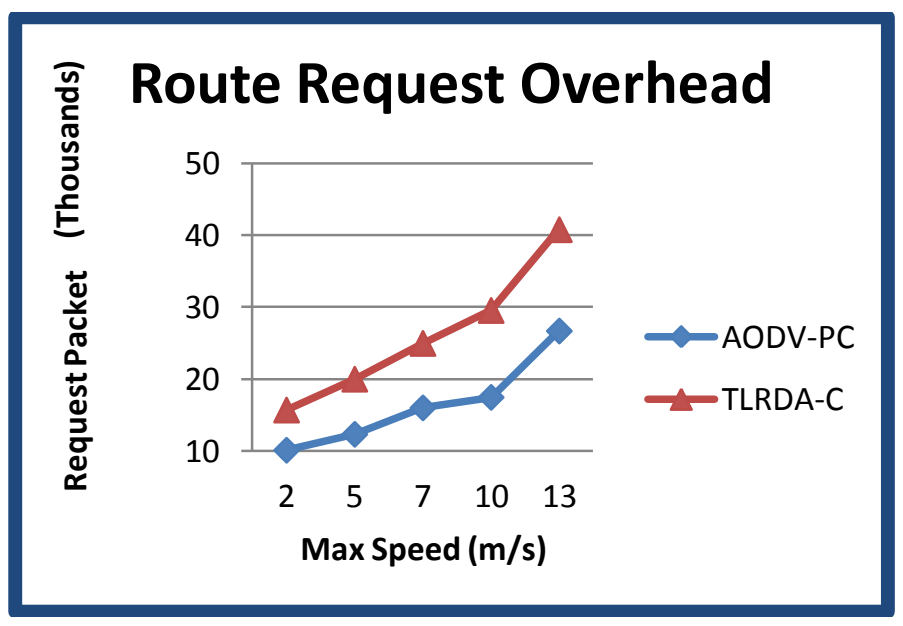

Fig.10. Route request overhead versus maximum speed

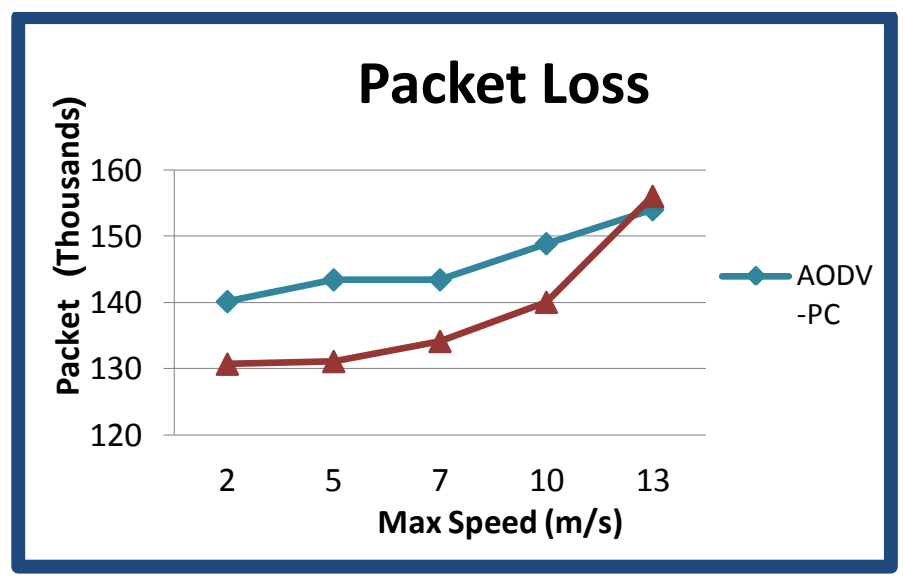

Fig.11. Packet loss versus maximum speed

\section{CONCLUSION}

This paper suggests new chasing packet technique used with standard On Demand Distance Vector protocol (AODV). A simulation and analysis for the proposed scheme are presented.
Some simulation experiments have been carried out to study AODV-PC and compare its performance with TLRDA$\mathrm{C}$ algorithm that uses chase packet concept. The simulation environments have considered different network scenarios with different parameters e.g. network density, and maximum speed under RWP model.

Simulation performance results show that that AODV-PC outperforms TLRDA-C in terms of the success rate of the catching process, end-to-end delay, route request latency. Due to avoiding the delay added to the route request when it reach to beyond-neighborhood region AODV-PC achieves better end-to-end delay when varying network density.

Furthermore, the route request latency improvement was up to $30 \%$. Despite the both algorithms have approximately equal results in term of packet loss; AODV-PC is less congests due to the reduction in the overhead caused by broadcasting the route request after the route is found.

\section{REFERENCES}

[1] Amera H. Al-Amery. Anew Routing Discovery Algorithm Using Parallel Chase Packets [dissertation]. Jordan University Of Science and Technology; 2010.

[2] Mznah A. Al-Rodhaan. Traffic Locality Oriented Route Discovery Algorithms for Mobile Ad Hoc Networks [dissertation]. University of Glasgow; 2009.

[3] Krishna G. Routing Protocols in Mobile Ad Hoc Networks [dissertation]. Sweden, Umea University; 2006 June.

[4] Peter L. Analysis of Routing Algorithms for Mobile Ad Hoc Networks [dissertation]. Chalmers University of Technology, Department of Computer Engineering, Gothenburg; 2002

[5] M. Aminu, M. Ould-Khaoua, L. M. Mackenzie and J. Abdulai. Performance Evaluation of an Efficient Counter-Based Scheme for Mobile Ad Hoc Networks based on Realistic Mobility Model. Proceedings of IEEE International Conference on Signal Processing and Communications; 2009.

[6] Incheon Park, Jinguk Kim, Ida Pu. Blocking Expanding Ring Search Algorithm for Efficient Energy Consumption in Mobile Ad Hoc Networks. Third Annual Conference on Wireless On-demand Network Systems and Services .2006. 191-195.

[7] Young-Bae Ko and Nitin H. Vaidya. Location-Aided Routing (LAR) in Mobile Ad Hoc Networks. Proceedings of the 4th Annual ACM/IEEE International Conference on Mobile Computing and Networking (Mobicom_98), November 1998; 66-75. Dallas.

[8] Ming-Yee Iu. Selective Flooding in Ad Hoc Networks [dissertation]. University of Waterloo, Waterloo, Ontario, Canada, 2002.

[9] I. Eltahir. The Impact of Different Radio Propagation Models for Mobile Ad Hoc Networks (MANET). Proceedings of the 2nd International Conference on Wireless Broadband and Ultra Wideband . 2007. 3032.

[10] Royer. E .Perkins. Ad Hoc On Demand Distance Vector Routing. Proceedings of 2nd IEEE Workshop on Mobile Computing Systems and Applications; 1999.

[11] Najla Al-Nabhan, Bowu Zhang, Mznah Al-Rodhaan, Abdullah AlDhelaan: Two Connected Dominating Set Algorithms for Wireless Sensor Networks. WASA 2012: 705-713.

[12] Muneer Bani Yassein, B. Abed Al-Hameed, Constandinos X. Mavromoustakis: Adaptive counter-based broadcasting scheme in mobile ad hoc networks. HP-MOSys 2012:47-52.

[13] Marios C. Charalambous, Constandinos X. Mavromoustakis, Muneer Bani Yassein: A Resource Intensive Traffic-Aware Scheme for Clusterbased Energy Conservation in Wireless Devices. HPCC-ICESS 2012:879-884. 